\title{
Search for Gauginos and Gauge Mediated SUSY Breaking Scenarios at LEP
}

\author{
Gabriella Pásztor ${ }^{* \dagger}$ \\ University of California, Riverside \\ E-mail: Gabriella.Pasztorecern.ch
}

Data collected by the OPAL detector at LEP up to the highest energies of $209 \mathrm{GeV}$ are analyzed to search for evidence of supersymmetric (SUSY) particle production.

Chargino pair-production $\left(\mathrm{e}^{+} \mathrm{e}^{-} \rightarrow \tilde{\chi}_{1}^{+} \tilde{\chi}_{1}^{-}\right)$and neutralino associated production $\left(\mathrm{e}^{+} \mathrm{e}^{-} \rightarrow \tilde{\chi}_{1}^{0} \tilde{\chi}_{2}^{0}\right)$ are considered in models where supersymmetry breaking is mediated by gravitational interactions from the hidden sector to the visible sector of the Standard Model and SUSY particles. R-parity conservation is assumed. The focus of the searches is on topologies arising when scalar fermions are heavy and the lightest neutralino is the lightest SUSY particle (LSP).

Searches for topologies predicted by gauge-mediated SUSY breaking (GMSB) models are also discussed. In these models the LSP is the gravitino and the phenomenology is driven by the nature of the next-to-lightest SUSY particle (NLSP) which is either the lightest neutralino, stau or mass degenerate sleptons. As the NLSP decay length is effectively unconstrained, all possible values are considered and results independent of the NLSP lifetime are presented for all relevant final states including direct NLSP pair-production and NLSP production via cascade decays of heavier SUSY particles.

None of the searches shows evidence for SUSY particle production. Cross-section limits are presented at the $95 \%$ confidence level both for direct NLSP production and, for the first time, also for cascade decays, providing the most general, almost model independent results. These are then interpreted in the framework of minimal theoretical models. Large areas of the accessible parameter space are excluded. In the super-gravity inspired Constrained MSSM (CMSSM) we derive the following limits for $m_{0}>500 \mathrm{GeV}, A_{0}=0$ and $\Delta M>5 \mathrm{GeV}$ on the lightest gaugino masses: $m_{\tilde{\chi}_{1}^{ \pm}}>101 \mathrm{GeV}, m_{\tilde{\chi}_{1}^{0}}>40 \mathrm{GeV}$ and $m_{\tilde{\chi}_{2}^{0}}>78 \mathrm{GeV}$, independent of $\tan \beta$. In the minimal GMSB model, the NLSP masses are constrained to be $m_{\tilde{\chi}_{1}^{0}}>53.5 \mathrm{GeV}, m_{\tilde{\tau}_{1}}>87.4 \mathrm{GeV}$ and $m_{\tilde{\ell}}>91.9 \mathrm{GeV}$ in the neutralino, stau and slepton co-NLSP scenarios, respectively.

International Europhysics Conference on High Energy Physics

July 21st - 27th 2005

Lisboa, Portugal

\footnotetext{
*Speaker.

† On leave of absence from KFKI RMKI, Budapest.
} 


\section{Introduction}

Supersymmetry, the best proposed solution to the problems of the Standard Model (SM), postulates the existence of a partner for each SM particle chirality state. The discovery of these superparners would be the most direct evidence for SUSY. Since SUSY particles are not observed with the same mass as their SM partners, SUSY must be broken. In the most widely investigated scenarios, it is assumed that SUSY is broken in some hidden sector of new particles and is communicated to the visible sector of SM and SUSY particles by gravity or gauge interactions.

We review the results of the searches for chargino and neutralino production [1] motivated by gravity-mediated SUSY breaking models, and then present a study of gauge-mediated SUSY breaking topologies [2] using the data collected by the OPAL detector at LEP up to the highest center-of-mass energies of $209 \mathrm{GeV}$.

\section{Searches for chargino and neutralino production}

The lightest charginos are expected to be pair-produced at LEP with a cross-section of typically a few pb. However, if the electron sneutrino is light, the cross-section decreases due to destructive interference between $s$-channel $\gamma / Z$ and $t$-channel $\tilde{v}_{\mathrm{e}}$ exchanges. If charginos are heavy, neutralino associated production may be the only SUSY signal at LEP.

The focus of the analyses [1] is on topologies arising when scalar fermions are heavy and the lightest neutralino is the LSP: $\mathrm{e}^{+} \mathrm{e}^{-} \rightarrow \tilde{\chi}_{1}^{+} \tilde{\chi}_{1}^{-} \rightarrow \mathrm{W}^{+*} \tilde{\chi}_{1}^{0} \mathrm{~W}^{-*} \tilde{\chi}_{1}^{0}$ and $\mathrm{e}^{+} \mathrm{e}^{-} \rightarrow \tilde{\chi}_{2}^{0} \tilde{\chi}_{1}^{0} \rightarrow\left(\mathrm{Z}^{0 *} \tilde{\chi}_{1}^{0}\right)\left(\tilde{\chi}_{1}^{0}\right)$. The kinematics strongly depend on the mass difference $\Delta M$ between $\tilde{\chi}_{1}^{ \pm}$(or $\tilde{\chi}_{2}^{0}$ ) and $\tilde{\chi}_{1}^{0}$.

Despite a small general excess in some of the channels, there is no indication for chargino or neutralino pair-production in the OPAL data. Upper cross-section limits are derived: $0.1 \mathrm{pb}$ is achieved almost up to the kinematic limit for leptonic and $0.3 \mathrm{pb}$ for semi-leptonic and hadronic final states in the chargino search, while $0.1 \mathrm{pb}$ for the hadronic final state of the neutralino search.

The results are interpreted in CMSSM where all gauginos are assumed to have a common mass $\left(m_{1 / 2}\right)$ at the GUT scale, implying a relation between their masses $\left(M_{i}\right)$ at the electroweak scale, and all sfermions to have a common mass $\left(m_{0}\right)$ at the SUSY breaking scale. Further parameters are: the pseudo-scalar Higgs boson mass $\left(m_{\mathrm{A}}\right)$, the ratio of the v.e.v. of the two Higgs field doublets $(\tan \beta)$, the mass mixing parameter of the Higgs field $(\mu)$ and the trilinear sfermion-Higgs coupling at the GUT scale $\left(A_{0}\right)$. As gaugino properties depend mainly on $M_{2}, \mu$ and $\tan \beta$, these parameters are scanned in detail, while the parameters determining the sfermion masses are fixed to $m_{0}=500 \mathrm{GeV}$ and $A_{0}=0$. The exclusion in the $\mu-M_{2}$ plane is shown for $\tan \beta=5$ on Figure 1 . The strongest exclusions are found for large $\tan \beta$. These exclusions can be turned into constraints on the masses as illustrated on Figure 1. For $\Delta M>5 \mathrm{GeV}$, we derive lower limits of 101,40 and $78 \mathrm{GeV}$ on the masses of $\tilde{\chi}_{1}^{ \pm}, \tilde{\chi}_{1}^{0}$ and $\tilde{\chi}_{2}^{0}$, respectively, independent of $\tan \beta$.

\section{Searches for GMSB topologies}

The main new feature of models with GMSB is a light gravitino LSP. The phenomenology is driven by the nature of the NLSP, which is either the lightest neutralino, stau or mass-degenerate 

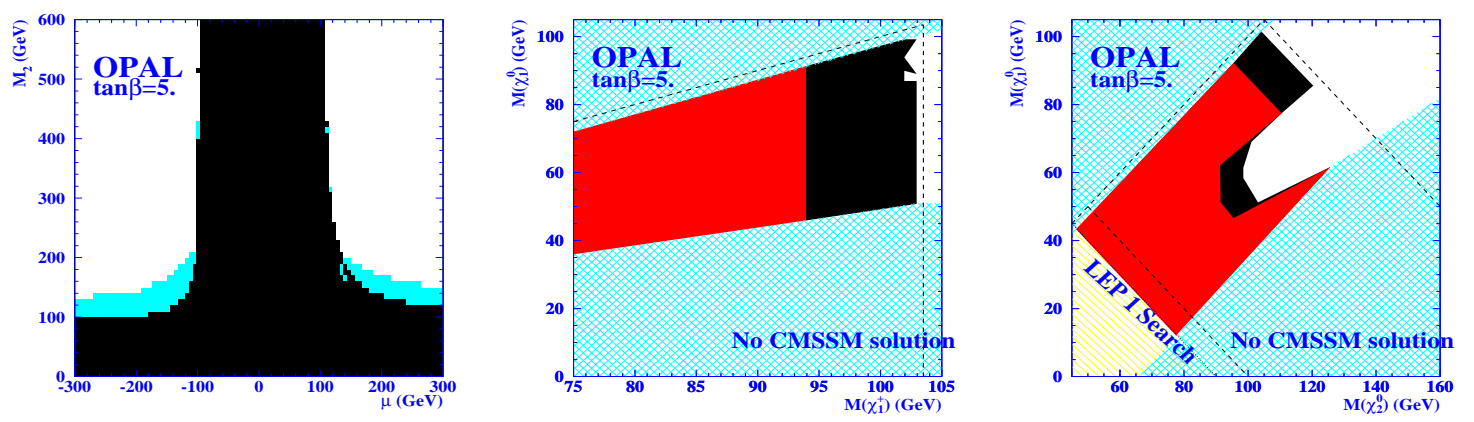

Figure 1: (left) Excluded region of the CMSSM parameter space (black) together with the kinematically allowed region (light blue/grey). (center) Excluded regions in the $\tilde{\chi}_{1}^{ \pm}-\tilde{\chi}_{1}^{0}$ mass plane by the $189 \mathrm{GeV}$ (red/grey) and the highest energy (black) OPAL data. (right) Excluded region in the $\tilde{\chi}_{2}^{0}-\tilde{\chi}_{1}^{0}$ mass plane.

sleptons. As the gravitino couples very weakly to heavier SUSY particles, those will decay typically to the NLSP which then decays via $\tilde{\chi}_{1}^{0} \rightarrow \gamma \tilde{\mathrm{G}}$ or $\tilde{\ell} \rightarrow \ell \tilde{\mathrm{G}}$. We study [2] all relevant final states: direct NLSP production and its appearance in the decay chain of heavier SUSY particles, like charginos, neutralinos and sleptons.

The minimal GMSB model introduces five new parameters and a sign: the SUSY breaking scale $(\sqrt{F})$, the SUSY particle mass scale $(\Lambda)$, the messenger mass $(M)$, the number of messenger sets $(N), \tan \beta$ and $\operatorname{sign}(\mu)$. As the decay length of the NLSP depends on $\sqrt{F}$ and is effectively unconstrained, NLSP decays inside and outside of the detector are searched for. With increasing decay length, the event signatures include: energetic leptons or photons and missing energy due to the undetected gravitino, tracks with large impact parameters, kinked tracks, or heavy long-lived charged particles. In total more than 14 different selections are developed to cover the GMSB topologies. The results are combined (with special attention to treat the overlaps among the many channels properly) to get lifetime independent results. To achieve a good description of the selection efficiencies over the whole mass and lifetime range at all center-of-mass energies, without generating an excessive number of Monte Carlo samples, an interpolating function is determined. On Fig 2 it is demonstrated how the different selections contribute to the signal detection efficiency as a function of the NLSP lifetime.

None of the searches shows evidence for SUSY particle production. To interpret the results, a detailed scan of the minimal GMSB parameter space is performed with the gravitino mass fixed to $2 \mathrm{eV}$, corresponding to $\sqrt{F} \approx 100 \mathrm{TeV}$, motivated by the requirement that the branching ratio of the next-to-NLSP to the gravitino is small. If that is fulfilled, the cross-sections and branching ratios do not depend on the gravitino mass. One should note that $\sqrt{F}$ can be eliminated from the scan as all limits are computed independent of the NLSP lifetime, and $\sqrt{F}$ has no significant effect on other particle masses.

"Model independent" cross-section limits are derived for each topology as a function of the NLSP lifetime. For direct NLSP decays, this is done by taking the worst limit for a given NLSP mass from the generated GMSB parameter scan points. For cascade channels, the cross-section evolution is assumed to be $\beta / s$ for spin- $1 / 2$ and $\beta^{3} / s$ for scalar SUSY particles, respectively, and the highest bound for all intermediate particle masses is retained. The maximum limit valid for all lifetimes is then quoted as the "lifetime independent" cross-section limit. In the neutralino NLSP scenario this is typically better than $0.04 \mathrm{pb}$ for direct NLSP production, $0.1 \mathrm{pb}$ for selectron and 

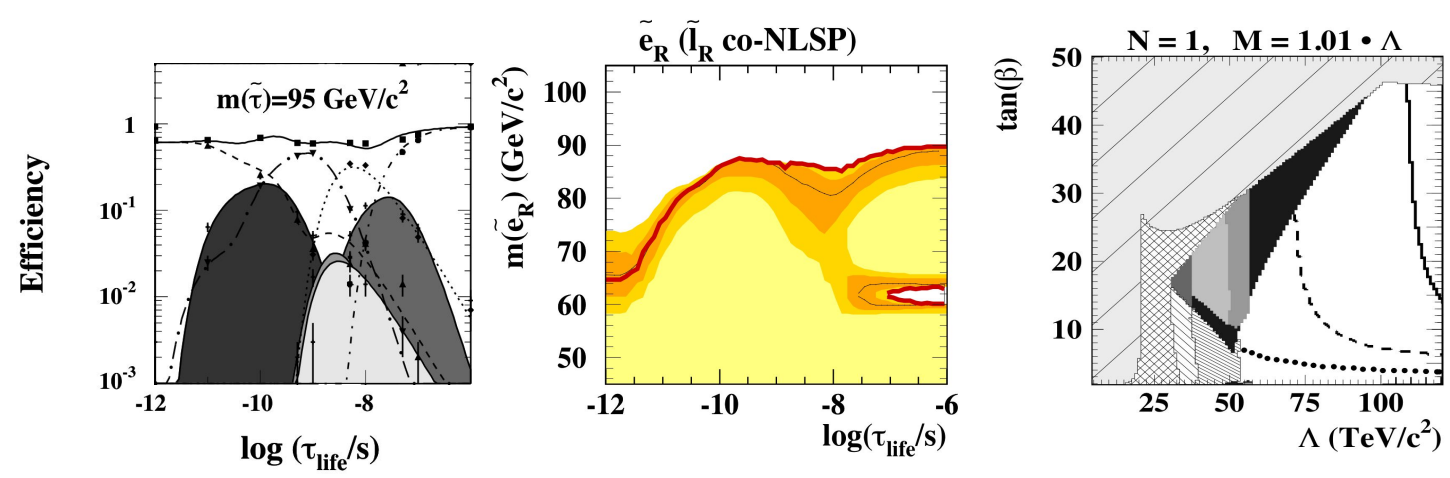

Figure 2: (left) Efficiencies for stau pair-production at $\sqrt{s}=208 \mathrm{GeV}$. The symbols represent the efficiencies for ten simulated lifetimes while the curves show the interpolating efficiency functions of the searches for promptly decaying staus (dashed), large impact parameters (long dash-dotted), kinks (dotted) and stable staus (dash-dotted) together with the overlap efficiencies (filled areas). The total efficiency is shown by full line. (center) Observed (thick red/dark grey) and expected (thin black) lower mass limits for pairproduced selectrons in the slepton co-NLSP scenario. The $68 \%$ and $95 \%$ probability intervals are shown by orange/grey shades. (right) Regions in the $\Lambda-\tan \beta$ plane excluded by the different searches in the stau and slepton co-NLSP scenarios (direct NLSP production - black, chargino - dark grey, neutralino grey, selectron and smuon production with stau NLSP - light grey) and in the neutralino NLSP scenario (neutralino production - dense hatched, chargino - hatched). The LEP1 search regions is cross-hatched and the theoretically not allowed region is grey with sparse hatching. The constraint from the LEP combined Higgs limit of $m_{\mathrm{H}}>114.4 \mathrm{GeV}$ is also indicated (full line) together with large effect weakening it from theoretical (dashed) plus top quark mass (dotted) uncertainities.

smuon production, $0.2 \mathrm{pb}$ for stau production and $0.3 \mathrm{pb}$ for chargino production. In the stau and slepton co-NLSP scenarios, the limit on direct NLSP production is $0.05 \mathrm{pb}$ for smuons, $0.1 \mathrm{pb}$ for selectrons and staus. For the cascade decays the bounds are typically better than $0.1 \mathrm{pb}$ for neutralino, 0.2 for chargino and in the stau NLSP scenario 0.4 for selectron and smuon production.

The cross-section limits can be turned into constraints on the NLSP mass. For sleptons, the lowest mass limits are found for very short lifetimes, except for selectrons, shown in Figure 2, where searches using $\mathrm{d} E / \mathrm{d} x$ measurements loose efficiency for particles with momenta around 65 $\mathrm{GeV}$. The lifetime independent limits are $m_{\tilde{\mathrm{e}}_{\mathrm{R}}}>60.1 \mathrm{GeV}, m_{\tilde{\mu}_{\mathrm{R}}}>93.7 \mathrm{GeV}$ and $m_{\tilde{\tau}_{1}}>87.4 \mathrm{GeV}$. The limit on the stau mass is the same in the stau and the slepton co-NLSP scenarios. In the slepton co-NLSP scenario, the best limit can be used to derive a universal limit on the slepton masses $m_{\tilde{\ell}}=m_{\tilde{\mu}_{\mathrm{R}}}-m_{\tau}>91.9 \mathrm{GeV}$, where by definition the mass differences between the different slepton flavors are smaller than the lepton masses. For neutralino NLSP, no lifetime independent NLSP mass limit can be set directly. For short lifetimes $\left(\tau<10^{-9} \mathrm{~s}\right)$ a mass limit of $96.8 \mathrm{GeV}$ is derived.

The GMSB parameter space can also be constrained by our results as shown in Figure 2 for $N=1, M=1.01 \cdot \Lambda$ and $\operatorname{sign}(\mu)>0$. The universal SUSY mass scale is $\Lambda>40,27,21,17,15 \mathrm{TeV}$ for messenger indices $N=1,2,3,4,5$, independent of $M, \tan \beta, \operatorname{sign}(\mu)$ and the NLSP lifetime $(\sqrt{F})$. The constraints on $\Lambda$ imply lower limits on the neutralino mass in the neutralino NLSP scenario: $m_{\tilde{\chi}_{1}^{0}}>53.5 \mathrm{GeV}$ for $\mathrm{N}=1$ and $m_{\tilde{\chi}_{1}^{0}}>94.0 \mathrm{GeV}$ for $\mathrm{N}=5$, independent of the lifetime.

\section{References}

[1] The OPAL Collaboration, G. Abbiendi et al., Eur. Phys. J. C35 (2004) 1.

[2] The OPAL Collaboration, G. Abbiendi et al., CERN-EP-PH-2005-025, submitted to Eur. Phys. J. C. 